\title{
iCAS: Imposed Automatic Selection and Localization of Complete Active Spaces
}

\author{
Yibo Lei, ${ }^{\dagger}$ Bingbing Suo, ${ }^{\ddagger}$ and Wenjian Liu*, II \\ tKey Laboratory of Synthetic and Natural Functional Molecule of the Ministry of Education, \\ College of Chemistry \& Materials Science, Shaanxi key Laboratory of Physico-Inorganic \\ Chemistry, Northwest University, Xi'an 710127, Shaanxi, P. R. China \\ $\ddagger$ Institute of Modern Physics, Northwest University, and Shaanxi Key Laboratory for Theoretical \\ Physics Frontiers, Xi'an 710127, Shaanxi, P. R. China \\ IQQingdao Institute for Theoretical and Computational Sciences, Institute of Frontier and \\ Interdisciplinary Science, Shandong University, Qingdao, Shandong 266237, P. R. China \\ E-mail: liuwj@sdu.edu.cn
}


Table S1: Cartesian coordinates of the singlet ground state of nonsymmetric ethylene

\begin{tabular}{cccc}
\hline \hline Atom & $\mathrm{X}$ & $\mathrm{Y}$ & $\mathrm{Z}$ \\
\hline $\mathrm{C}$ & -0.02961 & 0.022675 & 0.634743 \\
$\mathrm{C}$ & -0.02961 & 0.022675 & -0.70106 \\
$\mathrm{H}$ & 0.29128 & -0.72086 & 1.221416 \\
$\mathrm{H}$ & -0.85487 & -0.31451 & 1.284346 \\
$\mathrm{H}$ & 0.312949 & 0.838548 & -1.33754 \\
$\mathrm{H}$ & -0.90811 & 0.142641 & -1.3521 \\
\hline \hline
\end{tabular}




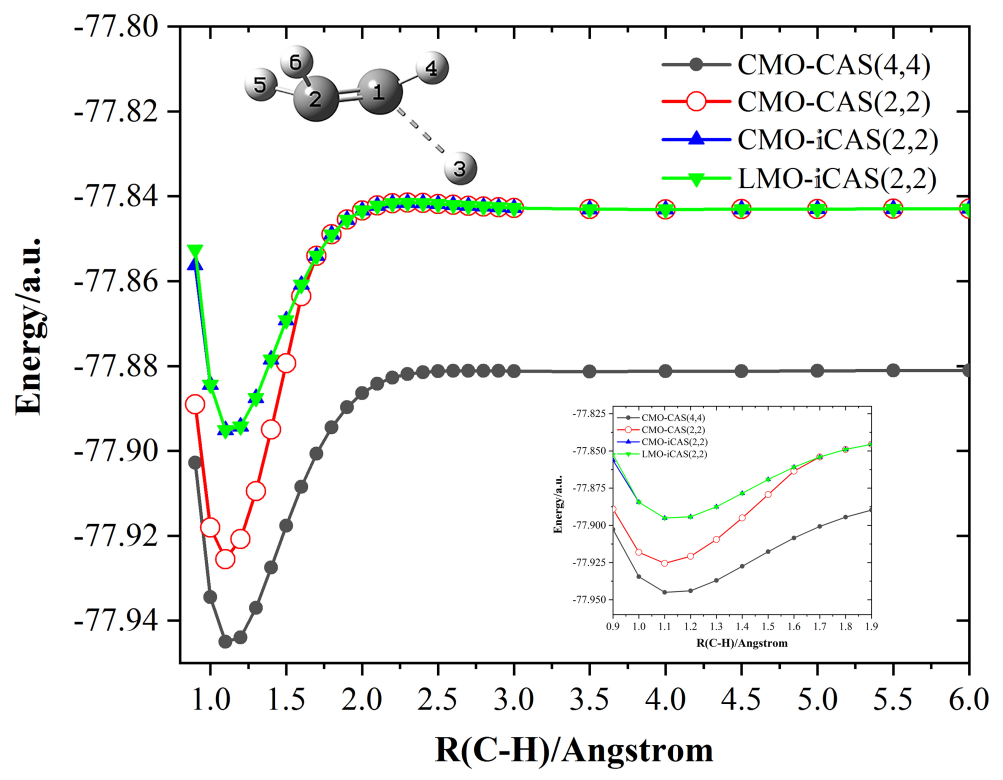

Figure S1: Potential energy curves for the breaking of single $\mathrm{C}-\mathrm{H}$ bond of nonsymmetric ethylene. 


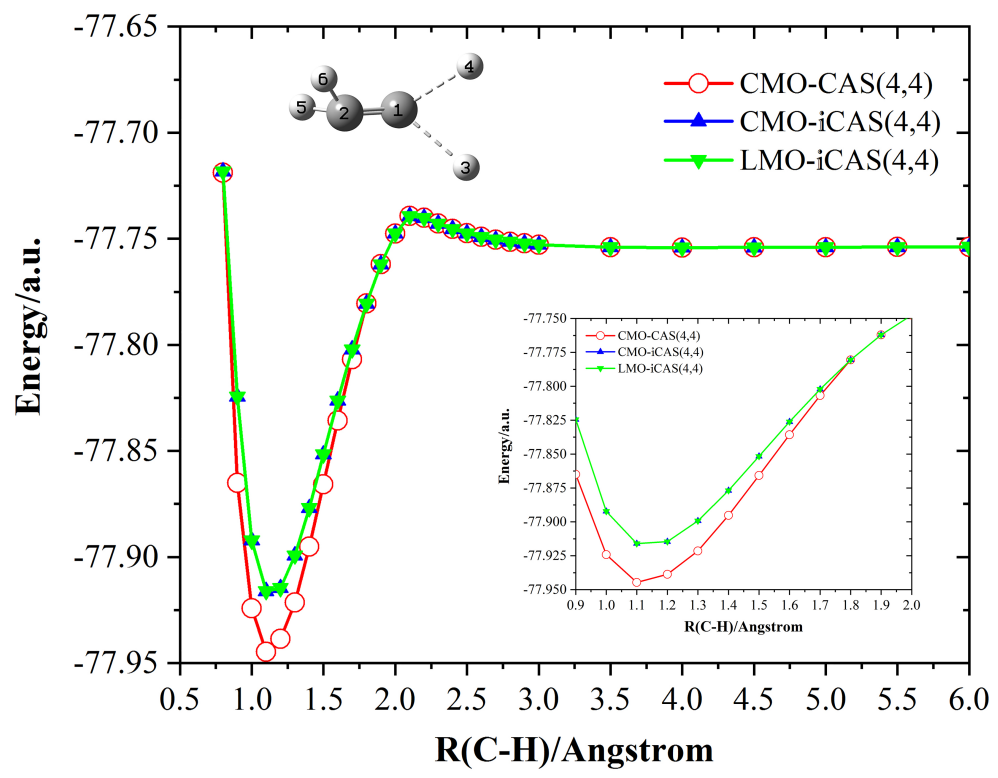

Figure S2: Potential energy curves for the simultaneous breaking of the two terminal $\mathrm{C}-\mathrm{H}$ bonds of nonsymmetric ethylene.

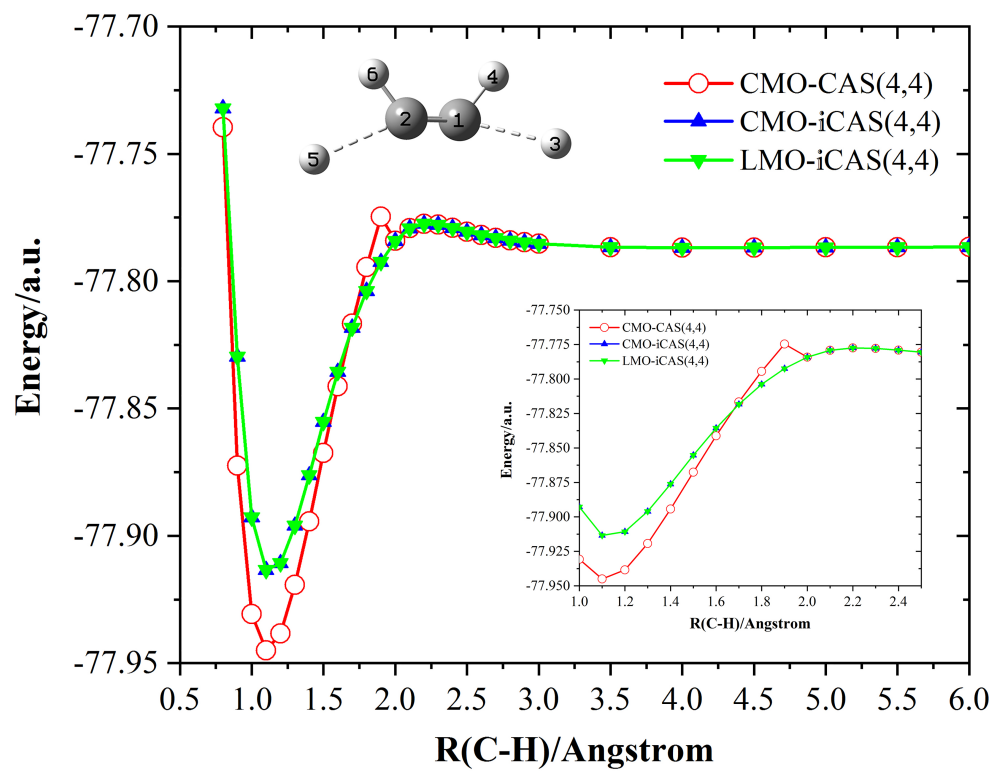

Figure S3: Potential energy curves for the simultaneous breaking of the two cis $\mathrm{C}-\mathrm{H}$ bonds of nonsymmetric ethylene. The step at $1.9 \AA$ on the CMO-CAS $(4,4)$ PEC is due to the swap of $\pi$ and $\sigma$ orbitals across this point. 


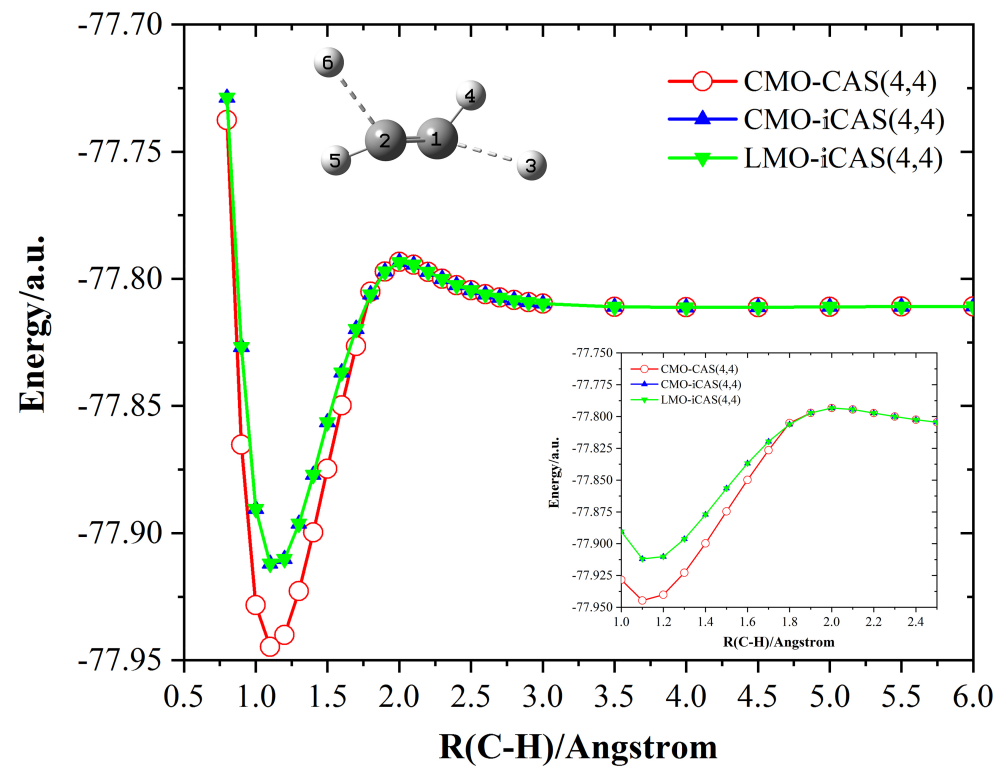

Figure S4: Potential energy curves for the simultaneous breaking of the two trans $\mathrm{C}-\mathrm{H}$ bonds of nonsymmetric ethylene.

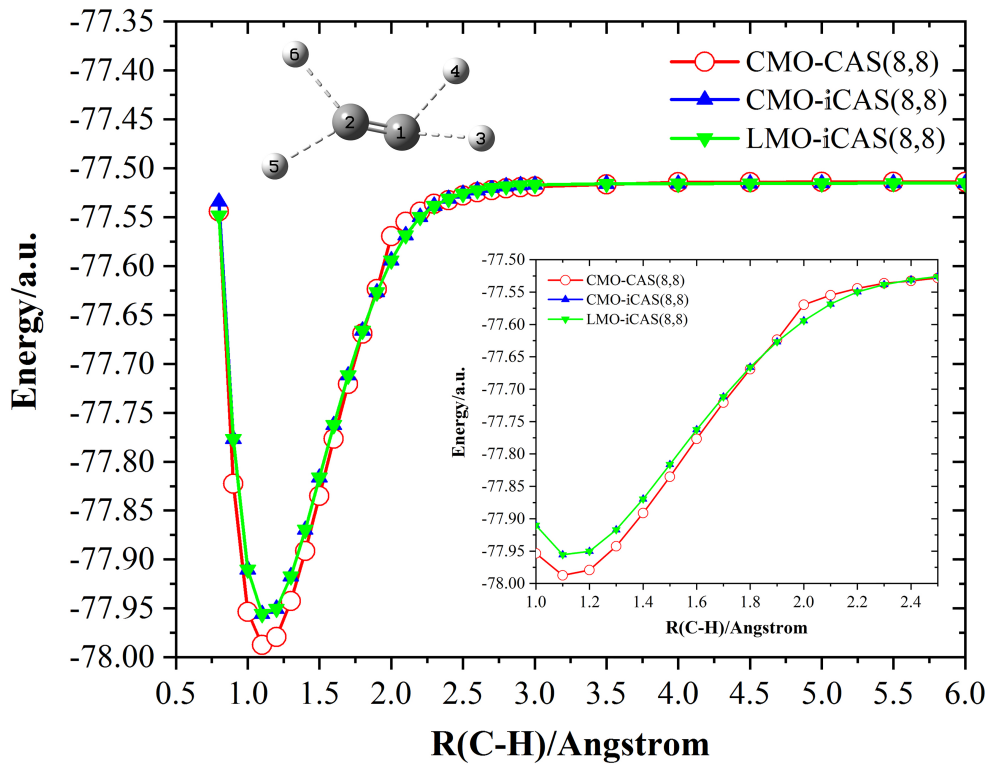

Figure S5: Potential energy curves for the simultaneous breaking of four $\mathrm{C}-\mathrm{H}$ bonds of nonsymmetric ethylene. 
Table S2: Cartesian coordinates of the singlet ground state of $\mathrm{Co}^{\mathrm{III}}$ (diiminato)(NPh) optimized by sf-X2C-PBE0/def2-SVP

\begin{tabular}{|c|c|c|c|}
\hline$\overline{\overline{\text { Atom }}}$ & $\bar{X}$ & $\bar{Y}$ & $\bar{Z}$ \\
\hline $\mathrm{C}$ & -1.218831 & -3.135692 & -0.002759 \\
\hline $\mathrm{C}$ & 1.217988 & -3.135942 & -0.002751 \\
\hline $\mathrm{C}$ & -0.000459 & -3.812911 & 0.000710 \\
\hline Co & -0.000134 & -0.530870 & -0.031033 \\
\hline $\mathrm{N}$ & -1.338147 & -1.817391 & -0.016547 \\
\hline $\mathrm{N}$ & 1.337626 & -1.817664 & -0.016533 \\
\hline $\mathrm{H}$ & -0.000583 & -4.902348 & 0.009191 \\
\hline $\mathrm{H}$ & 2.146026 & -3.724383 & 0.006035 \\
\hline $\mathrm{H}$ & -2.146950 & -3.723920 & 0.006015 \\
\hline $\mathrm{N}$ & 0.000020 & 1.079122 & -0.025853 \\
\hline $\mathrm{C}$ & 0.000047 & 2.435452 & -0.010026 \\
\hline C & -1.217526 & 3.154068 & -0.004335 \\
\hline $\mathrm{C}$ & 1.217745 & 3.154005 & -0.004341 \\
\hline $\mathrm{C}$ & -1.210817 & 4.542604 & 0.003086 \\
\hline $\mathrm{H}$ & -2.150879 & 2.586230 & -0.007495 \\
\hline $\mathrm{C}$ & 1.210987 & 4.542580 & 0.003082 \\
\hline $\mathrm{H}$ & 2.151034 & 2.586174 & -0.007508 \\
\hline $\mathrm{C}$ & 0.000124 & 5.240603 & 0.005892 \\
\hline $\mathrm{H}$ & -2.155886 & 5.091668 & 0.005697 \\
\hline $\mathrm{H}$ & 2.156087 & 5.091605 & 0.005693 \\
\hline $\mathrm{H}$ & 0.000123 & 6.333369 & 0.009667 \\
\hline C & 2.651609 & -1.274929 & -0.005340 \\
\hline $\mathrm{C}$ & 3.297151 & -1.021575 & 1.209114 \\
\hline $\mathrm{C}$ & 3.300503 & -0.975720 & -1.207431 \\
\hline $\mathrm{C}$ & 4.578864 & -0.475757 & 1.218627 \\
\hline $\mathrm{H}$ & 2.775925 & -1.246089 & 2.142370 \\
\hline $\mathrm{C}$ & 4.582794 & -0.431589 & -1.191907 \\
\hline $\mathrm{H}$ & 2.783485 & -1.166672 & -2.150397 \\
\hline $\mathrm{C}$ & 5.226203 & -0.178648 & 0.019515 \\
\hline $\mathrm{H}$ & 5.074836 & -0.279086 & 2.172420 \\
\hline $\mathrm{H}$ & 5.083174 & -0.202118 & -2.136041 \\
\hline $\mathrm{H}$ & 6.230939 & 0.250426 & 0.028987 \\
\hline C & -2.652078 & -1.274472 & -0.005348 \\
\hline $\mathrm{C}$ & -3.297590 & -1.021135 & 1.209082 \\
\hline $\mathrm{C}$ & -3.300932 & -0.975271 & -1.207418 \\
\hline $\mathrm{C}$ & -4.579313 & -0.475330 & 1.218610 \\
\hline $\mathrm{H}$ & -2.776354 & -1.245673 & 2.142370 \\
\hline $\mathrm{C}$ & -4.583249 & -0.431147 & -1.191907 \\
\hline $\mathrm{H}$ & -2.783913 & -1.166229 & -2.150411 \\
\hline $\mathrm{C}$ & -5.226628 & -0.178251 & 0.019514 \\
\hline $\mathrm{H}$ & -5.075292 & -0.278685 & 2.172421 \\
\hline $\mathrm{H}$ & -5.083621 & -0.201693 & -2.136047 \\
\hline $\mathrm{H}$ & -6.231429 & 0.250806 & 0.028981 \\
\hline
\end{tabular}


Table S3: Absolute overlaps between the seven doubly occupied pre-LMOs and all occupied molecular HF-LMOs for triplet $\mathrm{Co}^{\mathrm{III}}$ (diiminato)(NPh)

\begin{tabular}{ccccccccccc}
\hline \hline pre-LMO & active LMO & 1 & 2 & 3 & 4 & 5 & 6 & 7 & 8 & $\ldots$ b \\
\hline$b_{\|}$ & 0.984 & 0.000 & 0.000 & 0.000 & 0.000 & 0.000 & 0.000 & 0.000 & $\ldots$ \\
Co 3d $\mathrm{d}_{x z}$ & 0.000 & 0.999 & 0.000 & 0.000 & 0.000 & 0.000 & 0.000 & 0.000 & $\ldots$ \\
$\mathrm{Co} 3 \mathrm{~d}_{x^{2}-z^{2}}$ & 0.000 & 0.000 & 0.899 & 0.032 & 0.000 & 0.000 & 0.000 & 0.205 & $\ldots$ \\
$\mathrm{Co} 3 \mathrm{~d}_{y^{2}}$ & 0.000 & 0.000 & 0.032 & 0.881 & 0.000 & 0.000 & 0.000 & 0.333 & $\ldots$ \\
$\pi-1$ & 0.000 & 0.000 & 0.000 & 0.000 & 0.991 & 0.000 & 0.000 & 0.000 & $\ldots$ \\
$\pi-2$ & 0.000 & 0.000 & 0.000 & 0.000 & 0.000 & 0.990 & 0.000 & 0.000 & $\ldots$ \\
$\pi-3$ & 0.000 & 0.000 & 0.000 & 0.000 & 0.000 & 0.000 & 0.990 & 0.000 & $\ldots$ \\
& $0.969^{c}$ & $0.999^{c}$ & $0.810^{c}$ & $0.778^{c}$ & $0.982^{c}$ & $0.980^{c}$ & $0.980^{c}$ & $0.153^{\mathrm{c}}$ & $\ldots$ \\
\hline \hline
\end{tabular}

${ }^{\text {a }} b_{\|}$denotes $\operatorname{Co}\left(3 \mathrm{~d}_{x y}\right)-\mathrm{N}_{\text {imido }}\left(2 \mathrm{p}_{x}\right)$ in-plane $\pi ; \pi-1, \pi-2$ and $\pi-3$ correspond to localized $\pi$ of 1,3-propanediiminato.

${ }^{\mathrm{b}}$ Columns (core orbitals) with values smaller than 0.080 .

c Occupation number. 
Table S4: Absolute overlaps between the two singly occupied preLMOs and corresponding HF-LMOs for triplet $\mathrm{Co}^{\mathrm{III}}$ (diiminato)(NPh)

\begin{tabular}{ccc}
\hline $\begin{array}{c}\text { active LMO } \\
\text { pre-LMO }^{\mathrm{a}}\end{array}$ & 1 & 2 \\
\hline$b_{\perp}$ & 0.858 & 0.000 \\
$b_{\perp}^{*}$ & 0.000 & 0.997 \\
& $0.994^{\mathrm{b}}$ & $0.736^{\mathrm{b}}$ \\
\hline \hline
\end{tabular}

${ }^{\mathrm{a}} b_{\perp}\left(b_{\perp}^{*}\right)$ denotes $\operatorname{Co}\left(3 \mathrm{~d}_{y z}\right)-\mathrm{N}_{\text {imido }}\left(2 \mathrm{p}_{z}\right)$ out-of-plane

$\pi\left(\pi^{*}\right)$, in which $\operatorname{Co}\left(3 \mathrm{~d}_{y z}\right)$ and $\mathrm{N}_{\text {imido }}\left(2 \mathrm{p}_{z}\right)$ are the main component of $b_{\perp}$ and $b_{\perp}^{*}$, respectively.

${ }^{\mathrm{b}}$ Occupation number.

Table S5: Absolute overlaps between the eight unoccupied pre-LMOs and all unoccupied molecular HF-LMOs for triplet $\mathrm{Co}^{\mathrm{III}}$ (diiminato) $(\mathrm{NPh})$

\begin{tabular}{cccccccccccc}
\hline \hline pre-LMO & active LMO & 1 & 2 & 3 & 4 & 5 & 6 & 7 & 8 & 9 & $\ldots$ b \\
\hline$b_{\|}^{*}$ & 0.733 & 0.000 & 0.000 & 0.000 & 0.000 & 0.000 & 0.000 & 0.000 & 0.000 & $\ldots$ \\
$\pi^{*}-1$ & 0.000 & 0.870 & 0.084 & 0.000 & 0.000 & 0.000 & 0.000 & 0.000 & 0.000 & $\ldots$ \\
$\pi^{*}-2$ & 0.000 & 0.032 & 0.854 & 0.000 & 0.000 & 0.000 & 0.000 & 0.000 & 0.261 & $\ldots$ \\
Co $4 \mathrm{~d}_{y^{2}}$ & 0.000 & 0.000 & 0.000 & 0.771 & 0.000 & 0.000 & 0.000 & 0.000 & 0.000 & $\ldots$ \\
Co $4 \mathrm{~d}_{x^{2}-z^{2}}$ & 0.000 & 0.000 & 0.000 & 0.000 & 0.922 & 0.077 & 0.000 & 0.197 & 0.000 & $\ldots$ \\
${\mathrm{Co} 4 \mathrm{~d}_{x z}}_{\tilde{b}_{\perp}^{*}}$ & 0.000 & 0.000 & 0.000 & 0.000 & 0.000 & 0.920 & 0.000 & 0.000 & 0.063 & $\ldots$ \\
$\tilde{b}_{\|}^{*}$ & 0.435 & 0.000 & 0.000 & 0.000 & 0.000 & 0.000 & 0.871 & 0.000 & 0.000 & $\ldots$ \\
& 0.000 & 0.000 & 0.000 & 0.063 & 0.000 & 0.000 & 0.000 & 0.781 & 0.000 & $\ldots$ \\
\hline \hline
\end{tabular}

${ }^{a} b_{\|}^{*}$ denotes $\operatorname{Co}\left(3 \mathrm{~d}_{x y}\right)-\mathrm{N}_{\text {imido }}\left(2 \mathrm{p}_{x}\right)$ in-plane $\pi^{*} ; \tilde{b}_{\|}^{*}$ denotes $\operatorname{Co}\left(4 \mathrm{~d}_{x y}\right)-\mathrm{N}_{\text {imido }}\left(2 \mathrm{p}_{x}\right)$ in-plane $\pi^{*}$ with $\operatorname{Co}\left(4 \mathrm{~d}_{x y}\right)$ as the major; $\tilde{b}_{\perp}^{*}$ denotes $\operatorname{Co}\left(4 \mathrm{~d}_{y z}\right)-\mathrm{N}_{\text {imido }}\left(2 \mathrm{p}_{z}\right)$ out-of-plane $\pi^{*}$ with $\operatorname{Co}\left(4 \mathrm{~d}_{y z}\right)$ as the major; $\pi^{*}-1$ and $\pi^{*}-2$ correspond to localized $\pi^{*}$ of 1,3-propanediiminato.

${ }^{\mathrm{b}}$ Columns (virtual orbitals) with values smaller than 0.069 .

${ }^{\mathrm{c}}$ Occupation number. 
Table S6: Absolute overlaps between the six doubly occupied pre-LMOs and all occupied molecular HF-LMOs for quintet $\mathrm{Co}^{\mathrm{III}}$ (diiminato)(NPh)

\begin{tabular}{ccccccccc}
\hline \hline \begin{tabular}{c}
\hline active LMO \\
pre-LMO $^{\mathrm{a}}$
\end{tabular} & 1 & 2 & 3 & 4 & 5 & 6 & 7 & $\ldots \mathrm{b}$ \\
\hline$b_{\perp}$ & 0.993 & 0.000 & 0.005 & 0.003 & 0.002 & 0.008 & 0.001 & $\ldots$ \\
$b_{\|}$ & 0.002 & 0.986 & 0.000 & 0.002 & 0.001 & 0.012 & 0.000 & $\ldots$ \\
Co 3d $\mathrm{d}_{x^{2}-z^{2}}$ & 0.000 & 0.000 & 0.817 & 0.000 & 0.000 & 0.000 & 0.513 & $\ldots$ \\
$\pi-1$ & 0.003 & 0.000 & 0.000 & 0.990 & 0.001 & 0.012 & 0.000 & $\ldots$ \\
$\pi-2$ & 0.006 & 0.001 & 0.000 & 0.012 & 0.990 & 0.001 & 0.000 & $\ldots$ \\
$\pi-3$ & 0.006 & 0.000 & 0.001 & 0.012 & 0.012 & 0.990 & 0.001 & $\ldots$ \\
& $0.987^{c}$ & $0.973^{c}$ & $0.667^{c}$ & $0.980^{c}$ & $0.980^{c}$ & $0.981^{c}$ & $0.263^{c}$ & $\ldots$ \\
\hline \hline
\end{tabular}

${ }^{\mathrm{a}} b_{\|}$denotes $\operatorname{Co}\left(3 \mathrm{~d}_{x y}\right)-\mathrm{N}_{\text {imido }}\left(2 \mathrm{p}_{x}\right)$ in-plane $\pi ; b_{\perp}$ denotes $\operatorname{Co}\left(3 \mathrm{~d}_{y z}\right)-\mathrm{N}_{\text {imido }}\left(2 \mathrm{p}_{z}\right)$ out-of-plane $\pi ; \pi-1, \pi-2$ and $\pi-3$ correspond to localized $\pi$ of 1,3-propanediiminato.

${ }^{\mathrm{b}}$ Columns (core orbitals) with values smaller than 0.057 .

${ }^{c}$ Occupation number.

Table S7: Absolute overlaps between the four singly occupied pre-LMOs and corresponding HF-LMOs for quintet $\mathrm{Co}^{\mathrm{III}}$ (diiminato)(NPh)

\begin{tabular}{ccccc}
\hline \hline $\begin{array}{c}\text { active LMO } \\
\text { pre-LMO }^{\mathrm{a}}\end{array}$ & 1 & 2 & 3 & 4 \\
\hline${\mathrm{Co} 3 \mathrm{~d}_{x z}}^{\mathrm{Co} 3 \mathrm{~d}_{y^{2}}}$ & 1.000 & 0.001 & 0.000 & 0.001 \\
$b_{\perp}^{*}$ & 0.000 & 0.904 & 0.000 & 0.000 \\
$b_{\|}^{*}$ & 0.000 & 0.001 & 0.905 & 0.000 \\
& 0.000 & 0.000 & 0.001 & 0.882 \\
\hline \hline
\end{tabular}

\footnotetext{
a $b_{\|}^{*} \quad$ denotes $\operatorname{Co}\left(3 \mathrm{~d}_{x y}\right)-\mathrm{N}_{\text {imido }}\left(2 \mathrm{p}_{x}\right) \quad$ in-plane $\pi^{*} ; \quad b_{\perp}^{*}$ denotes $\operatorname{Co}\left(3 \mathrm{~d}_{y z}\right)-\mathrm{N}_{\text {imido }}\left(2 \mathrm{p}_{z}\right)$ out-of-plane $\pi^{*}$ in which $\mathrm{N}_{\text {imido }} 2 \mathrm{p}_{z}$ is major.

${ }^{\mathrm{b}}$ Occupation number.
} 
Table S8: Absolute overlaps between the seven unoccupied pre-LMOs and all unoccupied molecular HF-LMOs for quintet $\mathrm{Co}^{\mathrm{III}}$ (diiminato)(NPh)

\begin{tabular}{ccccccccccc}
\hline \hline pre-LMO & active LMO & 1 & 2 & 3 & 4 & 5 & 6 & 7 & 8 & $\ldots$ b \\
\hline$\pi^{*}-1$ & 0.869 & 0.023 & 0.007 & 0.043 & 0.003 & 0.002 & 0.008 & 0.003 & $\ldots$ \\
$\pi^{*}-2$ & 0.023 & 0.869 & 0.003 & 0.000 & 0.001 & 0.008 & 0.000 & 0.267 & $\ldots$ \\
Co $4 \mathrm{~d}_{x z}$ & 0.000 & 0.001 & 0.921 & 0.002 & 0.007 & 0.002 & 0.008 & 0.001 & $\ldots$ \\
Co $4 \mathrm{~d}_{x^{2}-z^{2}}$ & 0.000 & 0.002 & 0.002 & 0.868 & 0.001 & 0.010 & 0.001 & 0.005 & $\ldots$ \\
Co $4 \mathrm{~d}_{y^{2}}$ & 0.003 & 0.002 & 0.002 & 0.004 & 0.758 & 0.002 & 0.002 & 0.010 & $\ldots$ \\
$\tilde{b}_{\perp}^{*}$ & 0.000 & 0.001 & 0.007 & 0.092 & 0.032 & 0.936 & 0.006 & 0.000 & $\ldots$ \\
$\tilde{b}_{\|}^{*}$ & 0.001 & 0.002 & 0.041 & 0.004 & 0.032 & 0.023 & 0.726 & 0.000 & $\ldots$ \\
& $0.755^{c}$ & $0.755^{c}$ & $0.849^{c}$ & $0.764^{c}$ & $0.577^{c}$ & $0.877^{c}$ & $0.527^{c}$ & $0.071^{\mathrm{c}}$ & $\ldots$ \\
\hline \hline
\end{tabular}

\footnotetext{
a $\tilde{b}_{\|}^{*}$ denotes $\operatorname{Co}\left(4 \mathrm{~d}_{x y}\right)-\mathrm{N}_{\text {imido }}\left(2 \mathrm{p}_{x}\right)$ in-plane $\pi^{*}$ with $\operatorname{Co}\left(4 \mathrm{~d}_{x y}\right)$ as the major; $\tilde{b}_{\perp}^{*}$ denotes $\operatorname{Co}\left(4 \mathrm{~d}_{y z}\right)-\mathrm{N}_{\text {imido }}\left(2 \mathrm{p}_{z}\right)$ out-of-plane $\pi^{*}$ with $\operatorname{Co}\left(4 \mathrm{~d}_{y z}\right)$ as the major; $\pi^{*}-1$ and $\pi^{*}-2$ correspond to localized $\pi^{*}$ of 1,3-propanediiminato.

${ }^{\mathrm{b}}$ Columns (virtual orbitals) with values smaller than 0.072 .

c Occupation number.
} 
Table S9: Absolute overlaps between the initial and final LMO-SA2iCAS $(10,12)$ for triplet $\mathrm{Co}^{\text {III }}$ (diiminato)(NPh)

\begin{tabular}{|c|c|c|c|c|c|c|c|c|c|c|c|c|}
\hline initial $^{\mathrm{a}}$ final & 1 & 2 & 3 & 4 & 5 & 6 & 7 & 8 & 9 & 10 & 11 & 12 \\
\hline $\operatorname{Co~} 3 \mathrm{~d}_{y^{2}}$ & 0.927 & 0.000 & 0.000 & 0.000 & 0.000 & 0.000 & 0.000 & 0.000 & 0.008 & 0.000 & 0.000 & 0.000 \\
\hline $\operatorname{Co~} 3 \mathrm{~d}_{x^{2}-z^{2}}$ & 0.000 & 0.878 & 0.000 & 0.000 & 0.000 & 0.000 & 0.000 & 0.000 & 0.004 & 0.000 & 0.000 & 0.000 \\
\hline$b_{\|}$ & 0.000 & 0.000 & 0.977 & 0.000 & 0.000 & 0.000 & 0.001 & 0.004 & 0.000 & 0.000 & 0.000 & 0.000 \\
\hline$b_{\perp}$ & 0.000 & 0.005 & 0.000 & 0.998 & 0.000 & 0.000 & 0.000 & 0.000 & 0.001 & 0.001 & 0.000 & 0.001 \\
\hline $\operatorname{Co~} 3 d_{x z}$ & 0.000 & 0.000 & 0.000 & 0.000 & 0.996 & 0.000 & 0.000 & 0.000 & 0.000 & 0.000 & 0.001 & 0.000 \\
\hline$b_{\perp}^{*}$ & 0.000 & 0.005 & 0.000 & 0.000 & 0.000 & 0 & 0.000 & 0.000 & 0.000 & 0.001 & 0.000 & 0.001 \\
\hline$b_{\|}^{*}$ & 0.000 & 0.000 & 0.001 & 0.000 & 0.000 & 0.000 & 0.926 & 0.065 & 0.001 & 0.000 & 0.000 & 0.000 \\
\hline Co $4 \mathrm{~d}_{y^{2}}$ & 0.008 & 0.000 & 0.000 & 0.004 & 0.000 & 0.001 & 0.001 & 0.765 & 0.000 & 0.003 & 0.000 & 0.000 \\
\hline Co $4 d_{x^{2}-z^{2}}$ & 0.000 & 0.001 & 0.000 & 0.000 & 0.000 & 0.001 & 0.000 & 0.000 & 0.900 & 0.003 & 0.000 & 0.004 \\
\hline Co $4 \mathrm{~d}_{x z}$ & 0.000 & 0.000 & 0.000 & 0.000 & 0.001 & 0.000 & 0.000 & 0.001 & 0.000 & 0.921 & 0.000 & 0.000 \\
\hline$\tilde{b}_{\perp}^{*}$ & 0.000 & 0.001 & 0.000 & 0.000 & 0.000 & 0.001 & 0.000 & 0.000 & 0.000 & 0.004 & 0.924 & 0.000 \\
\hline$\tilde{b}_{\|}^{*}$ & 0.000 & 0.000 & 0.004 & 0.000 & 0.000 & 0.000 & 0.065 & 0.000 & 0.000 & 0.000 & 0.001 & 0.883 \\
\hline
\end{tabular}

${ }^{\mathrm{a}} b_{\|}\left(b_{\|}^{*}\right)$ denotes $\operatorname{Co}\left(3 \mathrm{~d}_{x y}\right)-\mathrm{N}_{\text {imido }}\left(2 \mathrm{p}_{x}\right)$ in-plane $\pi\left(\pi^{*}\right) ; b_{\perp}\left(b_{\perp}^{*}\right)$ denotes $\operatorname{Co}\left(3 \mathrm{~d}_{y z}\right)-\mathrm{N}_{\text {imido }}\left(2 \mathrm{p}_{z}\right)$ out-ofplane $\pi\left(\pi^{*}\right) ; \tilde{b}_{\|}^{*}$ denotes $\operatorname{Co}\left(4 \mathrm{~d}_{x y}\right)-\mathrm{N}_{\text {imido }}\left(2 \mathrm{p}_{x}\right)$ in-plane $\pi^{*}$ with $\operatorname{Co}\left(4 \mathrm{~d}_{x y}\right)$ as the major; $\tilde{b}_{\perp}^{*}$ denotes $\mathrm{Co}\left(4 \mathrm{~d}_{y z}\right)-\mathrm{N}_{\text {imido }}\left(2 \mathrm{p}_{z}\right)$ out-of-plane $\pi^{*}$ with $\mathrm{Co}\left(4 \mathrm{~d}_{y z}\right)$ as the major. 
Table S10: Absolute overlaps between the initial and final LMO-SA2iCAS $(10,12)$ for quintet $\mathrm{Co}^{\mathrm{III}}$ (diiminato)(NPh)

\begin{tabular}{|c|c|c|c|c|c|c|c|c|c|c|c|c|}
\hline initial $^{\mathrm{a}}$ final & 1 & 2 & 3 & 4 & 5 & 6 & 7 & 8 & 9 & 10 & 11 & 12 \\
\hline $\operatorname{Co~} 3 d_{x^{2}-z^{2}}$ & 0.805 & 0.000 & 0.000 & 0.000 & 0.000 & 0.000 & 0.000 & 0.000 & 0.000 & 0.001 & 0.000 & 0.000 \\
\hline$b_{\|}$ & 0.000 & 0.973 & 0.000 & 0.000 & 0.002 & 0.000 & 0.000 & 0.000 & 0.000 & 0.000 & 0.000 & 0.000 \\
\hline$b_{\perp}$ & 0.000 & 0.000 & 0.991 & 0.000 & 0.000 & 0.002 & 0.000 & 0.000 & 0.001 & 0.000 & 0.000 & 0.000 \\
\hline $\operatorname{Co~} 3 d_{y^{2}}$ & 0.000 & 0.000 & 0.000 & 1.000 & 0.000 & 0.000 & 0.000 & 0.000 & 0.000 & 0.001 & 0.000 & 0.004 \\
\hline Co $3 \mathrm{~d}_{x z}$ & 0.000 & 0.000 & 0.000 & 0.000 & 1.000 & 0.000 & 0.000 & 0.003 & 0.000 & 0.000 & 0.000 & 0.000 \\
\hline$b_{\perp}^{*}$ & 0.002 & 0.000 & 0.000 & 0.000 & 0.000 & 0.999 & 0.000 & 0.000 & 0.000 & 0.000 & 0.000 & 0.000 \\
\hline$b_{\|}^{*}$ & 0.000 & 0.002 & 0.000 & 0.000 & 0.000 & 0.000 & 0.999 & 0.000 & 0.000 & 0.000 & 0.006 & 0.000 \\
\hline Co $4 \mathrm{~d}_{y^{2}}$ & 0.000 & 0.000 & 0.000 & 0.000 & 0.000 & 0.000 & 0.004 & 0.821 & 0.000 & 0.008 & 0.000 & 0.001 \\
\hline Co $4 d_{x^{2}-z^{2}}$ & 0.000 & 0.000 & 0.000 & 0.001 & 0.000 & 0.000 & 0.001 & 0.000 & 0.910 & 0.000 & 0.001 & 0.008 \\
\hline Co $4 \mathrm{~d}_{x z}$ & 0.000 & 0.000 & 0.003 & 0.000 & 0.000 & 0.000 & 0.000 & 0.000 & 0.000 & 0.922 & 0.001 & 0.001 \\
\hline$\tilde{b}_{\perp}^{*}$ & 0.001 & 0.000 & 0.000 & 0.000 & 0.000 & 0.000 & 0.000 & 0.000 & 0.000 & 0.000 & 0.939 & 0.000 \\
\hline$\tilde{b}_{\|}^{*}$ & 0.000 & 0.000 & 0.000 & 0.000 & 0.006 & 0.000 & 0.000 & 0.001 & 0.000 & 0.001 & 0.000 & 0.833 \\
\hline
\end{tabular}

${ }^{\mathrm{a}} b_{\|}\left(b_{\|}^{*}\right)$ denotes $\operatorname{Co}\left(3 \mathrm{~d}_{x y}\right)-\mathrm{N}_{\text {imido }}\left(2 \mathrm{p}_{x}\right)$ in-plane $\pi\left(\pi^{*}\right) ; b_{\perp}\left(b_{\perp}^{*}\right)$ denotes $\operatorname{Co}\left(3 \mathrm{~d}_{y z}\right)-\mathrm{N}_{\text {imido }}\left(2 \mathrm{p}_{z}\right)$ out-ofplane $\pi\left(\pi^{*}\right) ; \tilde{b}_{\|}^{*}$ denotes $\operatorname{Co}\left(4 \mathrm{~d}_{x y}\right)-\mathrm{N}_{\text {imido }}\left(2 \mathrm{p}_{x}\right)$ in-plane $\pi^{*}$ with $\operatorname{Co}\left(4 \mathrm{~d}_{x y}\right)$ as the major; $\tilde{b}_{\perp}^{*}$ denotes $\mathrm{Co}\left(4 \mathrm{~d}_{y z}\right)-\mathrm{N}_{\text {imido }}\left(2 \mathrm{p}_{z}\right)$ out-of-plane $\pi^{*}$ with $\mathrm{Co}\left(4 \mathrm{~d}_{y z}\right)$ as the major. 


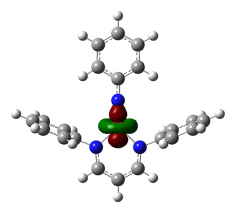

Co $3 \mathrm{~d} y^{2}$

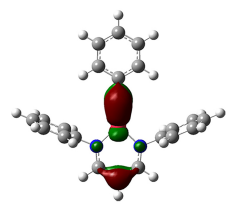

Co-N out-of-plane $\pi$

+ conjugated $\pi$

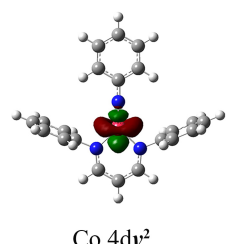

$\operatorname{Co} 4 \mathrm{~d} y^{2}$

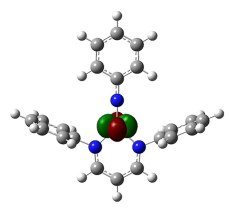

$\operatorname{Cos} 3 d x^{2}-z^{2}$

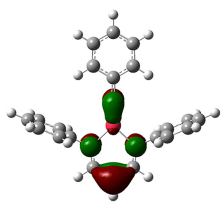

Co-N out-of-plane $\pi$

- conjugated $\pi$

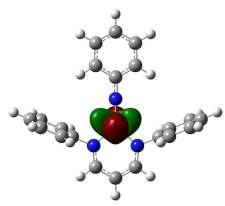

$\operatorname{Co} 4 \mathrm{~d} x^{2}-z^{2}$

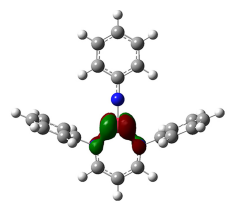

Co $3 \mathrm{~d} x \boldsymbol{z}$

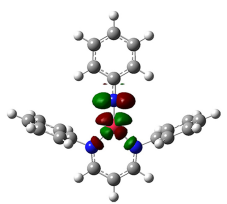

$\operatorname{Co}(3 \mathrm{~d})-\mathrm{N}$ in-plane $\pi^{*}$

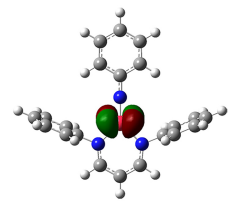

Co $4 \mathrm{~d} x z$

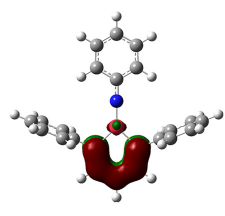

conjugated $\pi-1$

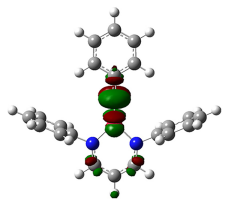

$\mathrm{Co}(3 \mathrm{~d})-\mathrm{N}$ out-of-plane $\pi^{*}$

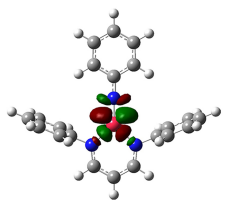

$\operatorname{Co}(4 d)-N$ in-plane $\pi^{*}$

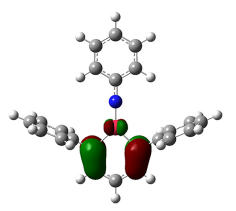

conjugated $\pi-2$

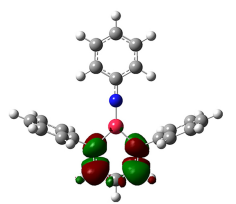

conjugated $\pi^{*}-1$

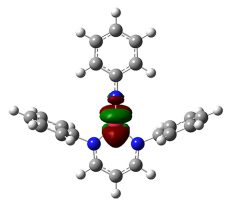

$\operatorname{Co}(4 \mathrm{~d})-\mathrm{N}$ out-of-plane $\pi^{*}$

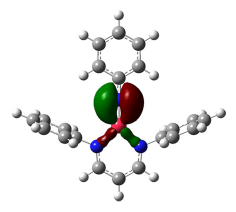

Co-N in-plane $\pi$

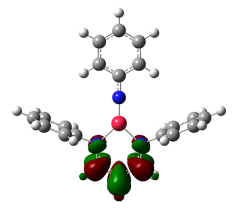

conjugated $\pi^{*}-2$

Figure S6: Automatically selected initial 17 active CMOs of singlet Co ${ }^{\mathrm{III}}$ (diiminato)(NPh). 


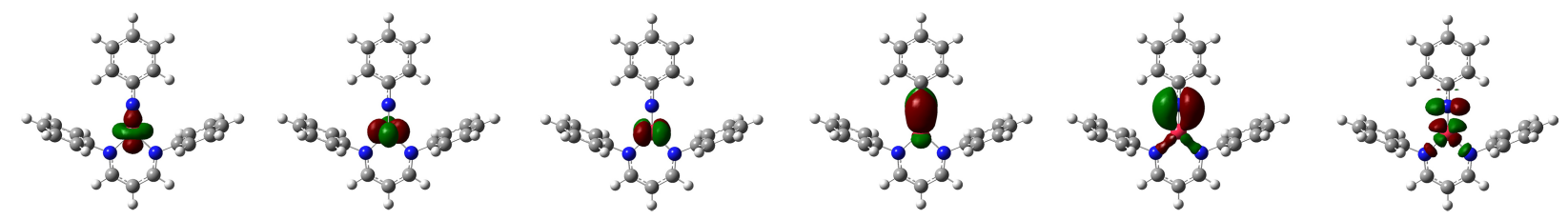

$\begin{array}{llllll}\text { Co } 3 \mathrm{~d} y^{2} & \text { Co } 3 \mathrm{~d} x^{2}-z^{2} & \text { Co } 3 \mathrm{~d} x z & \text { Co-N out-of-plane } \pi & \text { Co-N in-plane } \pi & \text { Co(3d)-N in-plane } \pi^{*}\end{array}$

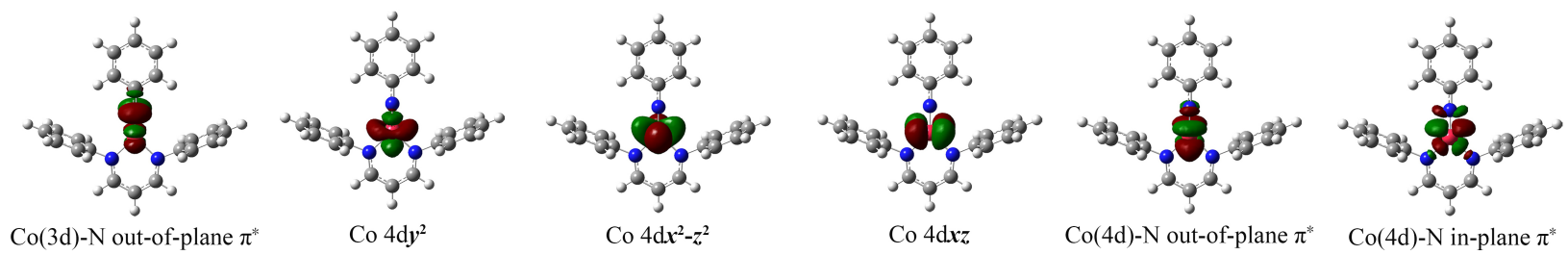

Figure S7: Initial CMOs of CMO-SA2-iCAS $(10,12)$ of singlet Co ${ }^{\text {III }}$ (diiminato)(NPh).

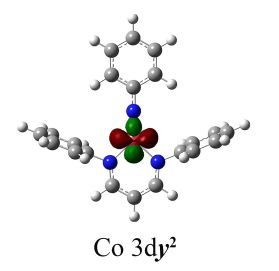

Co $3 \mathrm{~d} y^{2}$

(1.97)

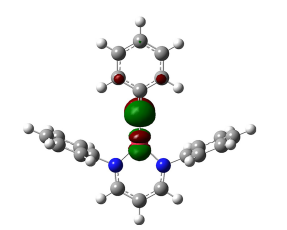

$\mathrm{Co}(3 \mathrm{~d})-\mathrm{N}$ out-of-plane $\pi^{*}$ $(0.68)$

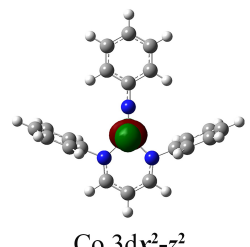

Co $3 \mathrm{~d} x^{2}-z^{2}$

(1.47)

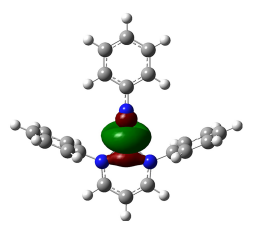

$\operatorname{Co} 4 \mathrm{~d} y^{2}$

(0.03)

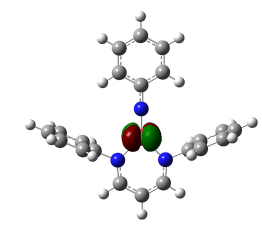

Co $3 \mathrm{~d} x z$

(1.96)

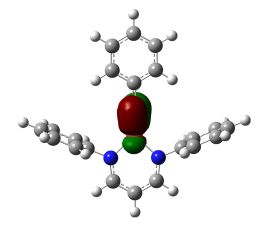

Co-N out-of-plane $\pi$

(1.82)

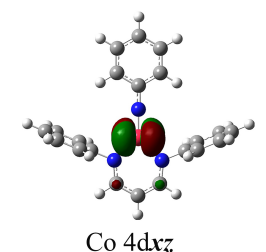

$(0.02)$

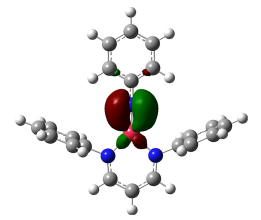

Co-N in-plane $\pi$

(1.87)

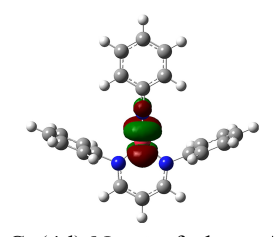

$\operatorname{Co}(4 \mathrm{~d})-\mathrm{N}$ out-of-plane $\pi^{*}$ $(0.01)$

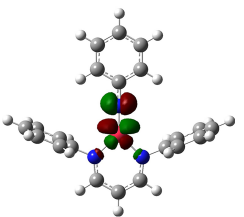

$\mathrm{Co}(3 \mathrm{~d})-\mathrm{N}$ in-plane $\pi^{*}$

(0.15)

Figure S8: Optimized CMOs of CMO-SA2-iCAS(10,12) of singlet Co ${ }^{\mathrm{III}}$ (diiminato)(NPh). Occupation numbers are shown in parentheses. 

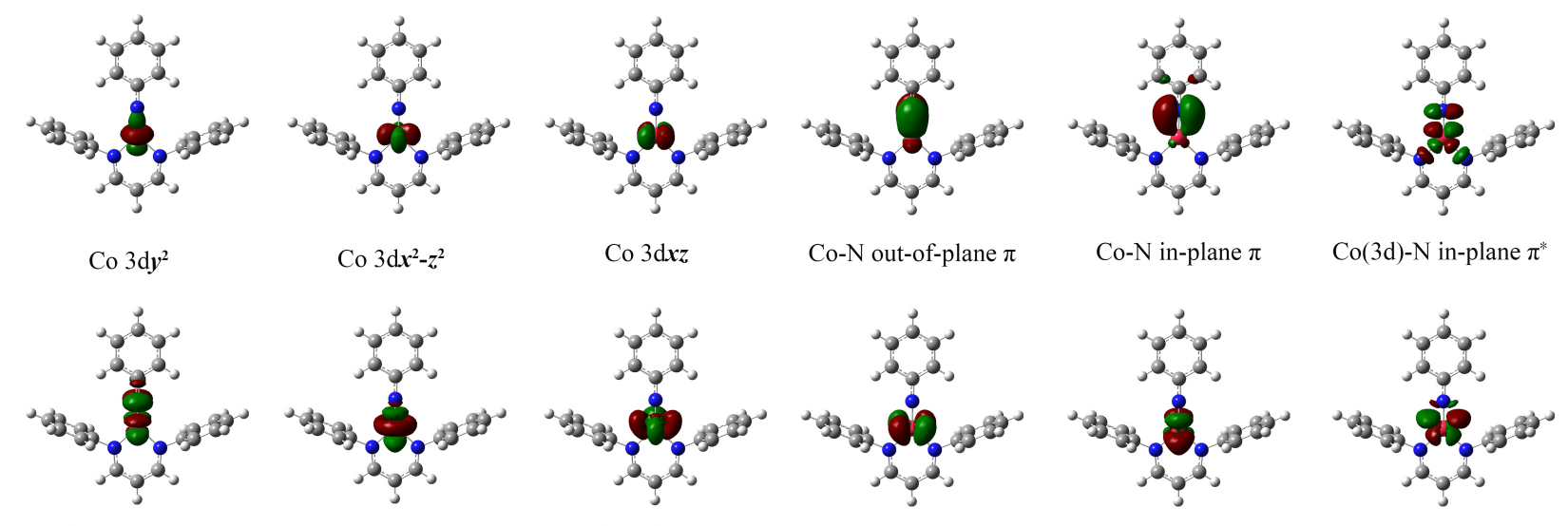

$\operatorname{Co}(3 \mathrm{~d})$-N out-of-plane $\pi^{*}$

Co $4 \mathrm{~d} y^{2}$

Co $4 \mathrm{~d} x^{2}-z^{2}$

Co $4 \mathrm{~d} x z$

Co(4d)-N out-of-plane $\pi^{*} \quad \operatorname{Co}(4 d)-N$ in-plane $\pi^{*}$

Figure S9: Initial LMOs of LMO-SA2-iCAS $(10,12)$ of singlet $\mathrm{Co}^{\mathrm{III}}$ (diiminato)(NPh).

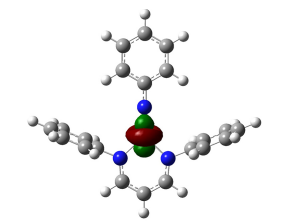

Co $3 \mathrm{~d} y^{2}$

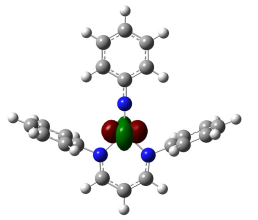

Co $3 \mathrm{~d} x^{2}-z^{2}$

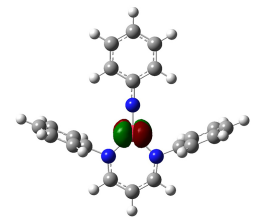

Co $3 \mathrm{~d} x z$

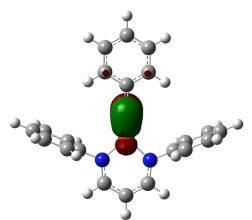

Co-N out-of-plane $\pi$

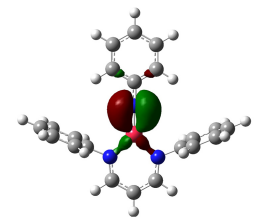

Co-N in-plane $\pi$

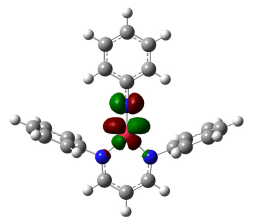

$\operatorname{Co}(3 \mathrm{~d})-\mathrm{N}$ in-plane $\pi^{*}$

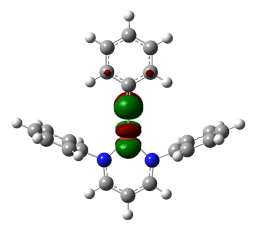

$\operatorname{Co}(3 d)-N$ out-of-plane $\pi^{*}$

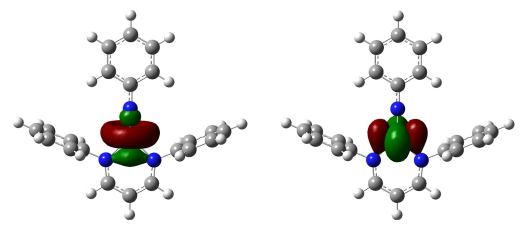

Co $4 \mathrm{~d} y^{2}$

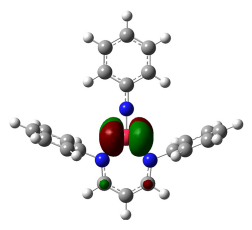

Co $4 \mathrm{~d} x z$

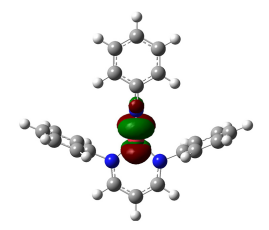

Co(4d)-N out-of-plane $\pi^{*}$

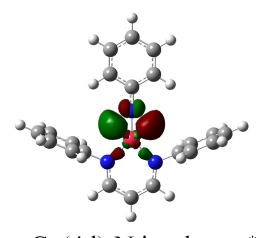

$\mathrm{Co}(4 \mathrm{~d})-\mathrm{N}$ in-plane $\pi^{*}$

Figure S10: Optimized LMOs of LMO-SA2-iCAS $(10,12)$ of singlet Co ${ }^{\mathrm{III}}$ (diiminato)(NPh). 


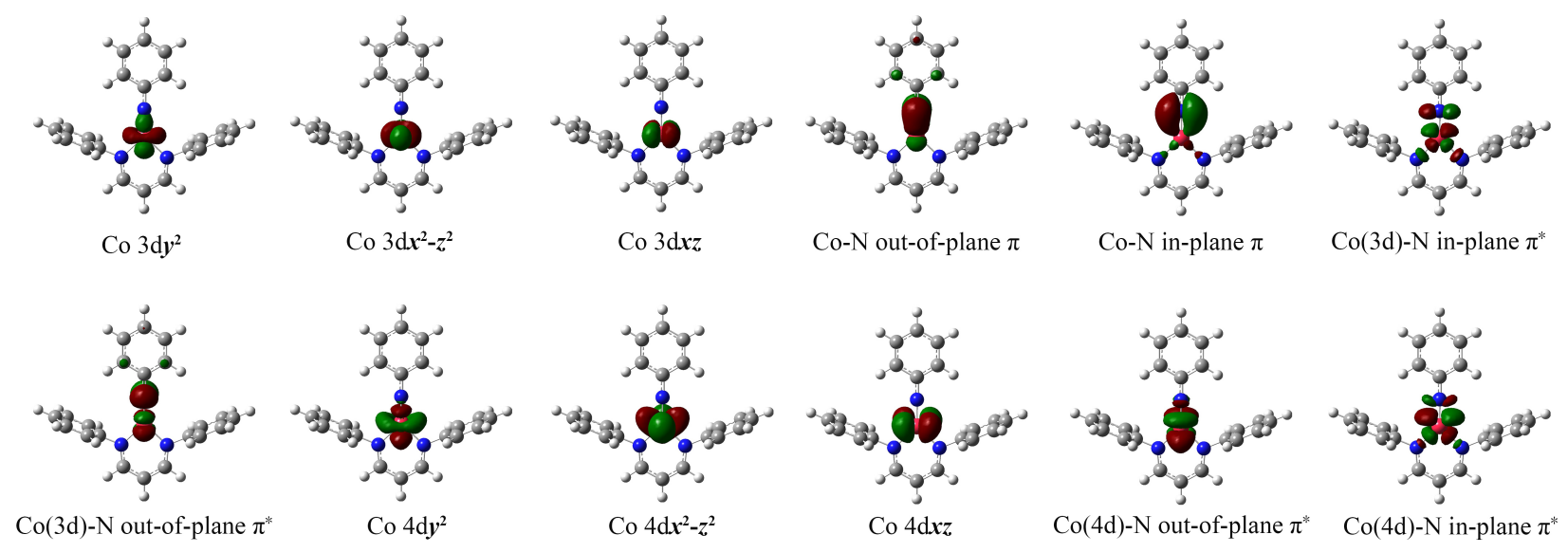

Figure S11: Initial CMOs of CMO-SA2-iCAS $(10,12)$ of triplet Co ${ }^{\text {III }}$ (diiminato)(NPh).

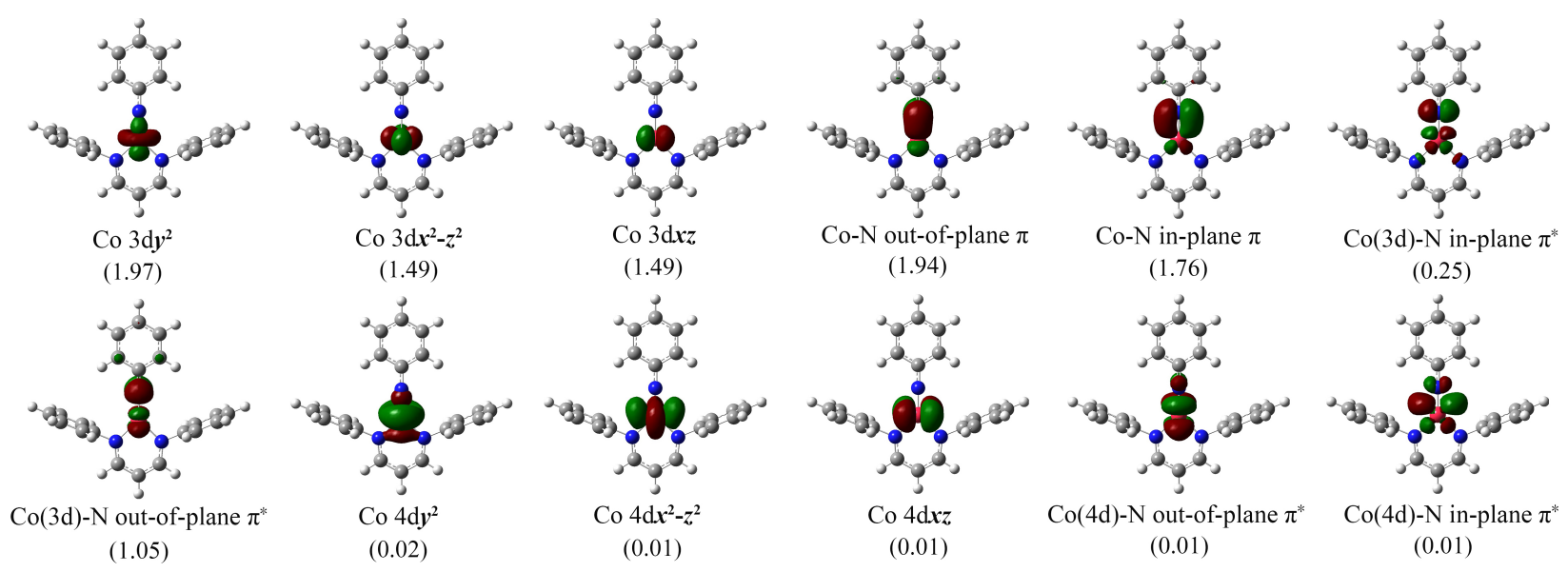

Figure S12: Optimized CMOs of CMO-SA2-iCAS $(10,12)$ of triplet Co ${ }^{\mathrm{III}}$ (diiminato)(NPh). Occupation numbers are shown in parentheses. 

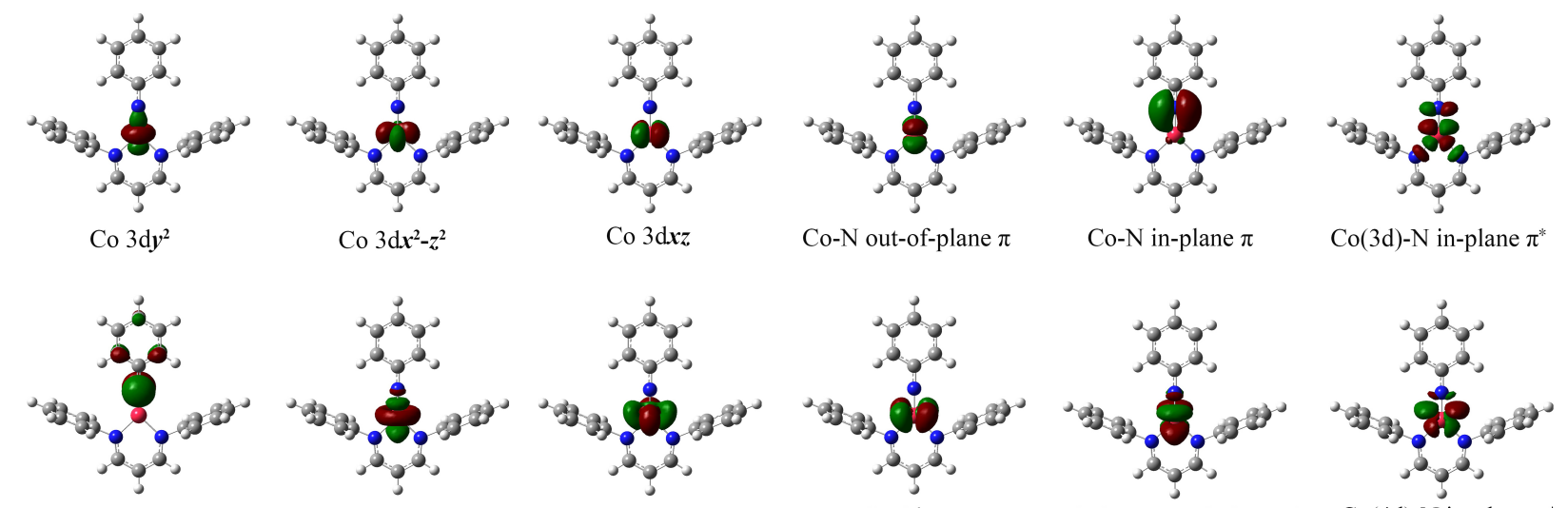

Co(3d)-N out-of-plane $\pi^{*}$

Co $4 \mathrm{~d} y^{2}$

Co $4 \mathrm{~d} x^{2}-z^{2}$

Co 4d $x z$

Co(4d)-N out-of-plane $\pi^{*}$

Co(4d)-N in-plane $\pi^{*}$

Figure S13: Initial LMOs of LMO-SA2-iCAS $(10,12)$ of triplet Co ${ }^{\text {III }}$ (diiminato)(NPh).

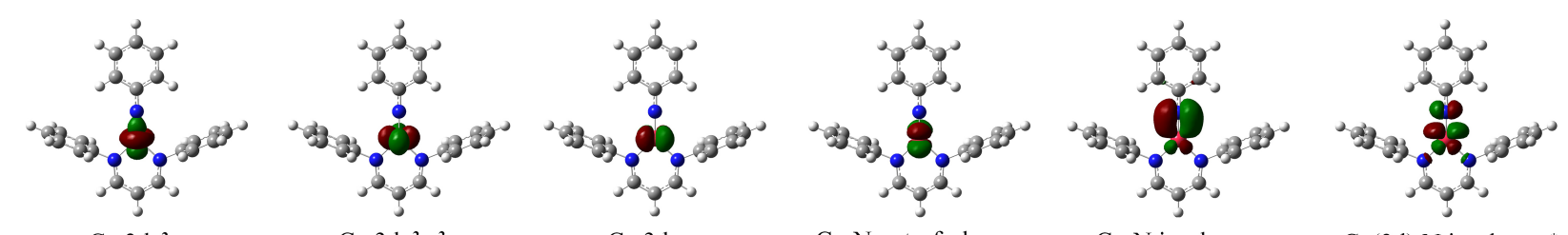

Co $3 \mathrm{~d} y^{2} \quad$ Co $3 \mathrm{~d} x^{2}-z^{2} \quad$ Co $3 \mathrm{~d} x z \quad$ Co-N out-of-plane $\pi \quad$ Co-N in-plane $\pi \quad$ Co(3d)-N in-plane $\pi^{*}$

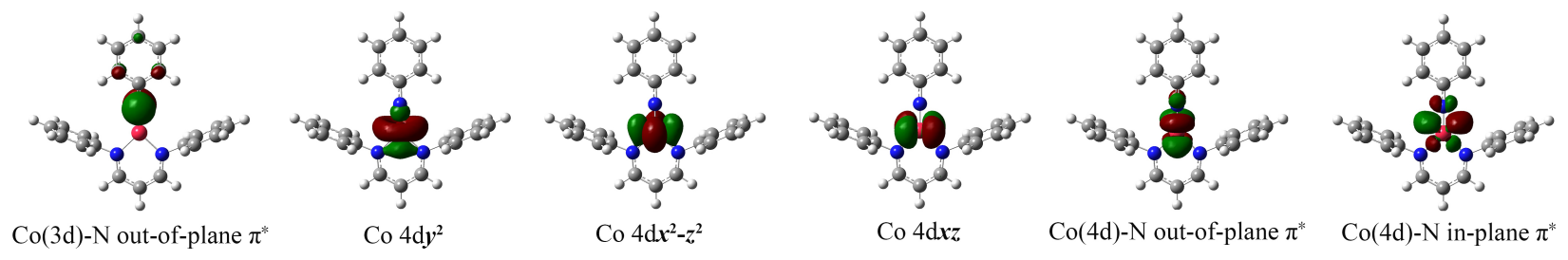

Figure S14: Optimized LMOs of LMO-SA2-iCAS(10,12) of triplet Co ${ }^{\text {III }}$ (diiminato)(NPh). 


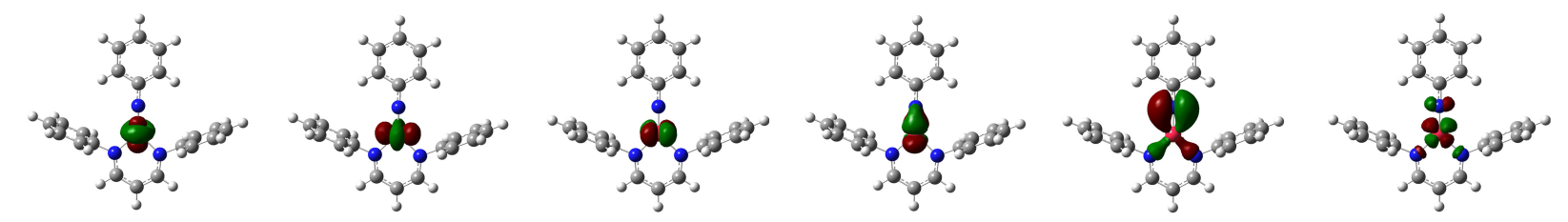

$\begin{array}{llllll}\text { Co } 3 \mathrm{~d} y^{2} & \text { Co } 3 \mathrm{~d} x^{2}-z^{2} & \text { Co } 3 \mathrm{~d} x z & \text { Co-N out-of-plane } \pi & \text { Co-N in-plane } \pi & \text { Co(3d)-N in-plane } \pi^{*}\end{array}$

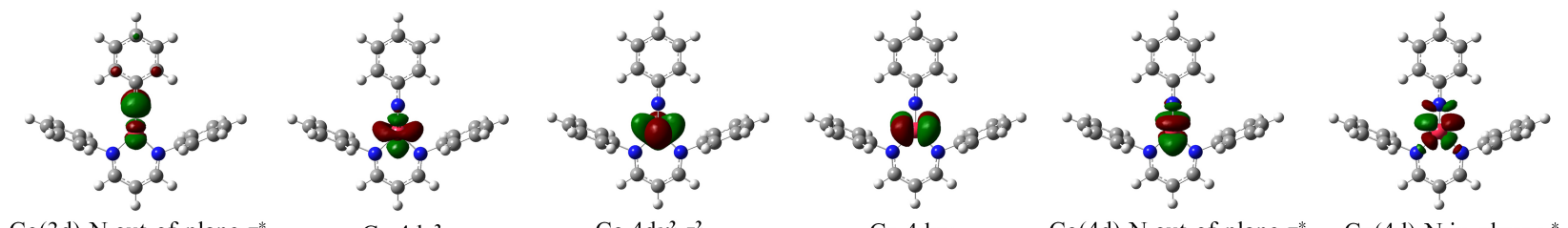

$\mathrm{Co}(3 \mathrm{~d})-\mathrm{N}$ out-of-plane $\pi^{*}$

Co $4 \mathrm{~d} y^{2}$

Co $4 \mathrm{~d} x^{2}-z^{2}$

$\operatorname{Co} 4 \mathrm{~d} x z$

$\mathrm{Co}(4 \mathrm{~d})-\mathrm{N}$ out-of-plane $\pi^{*}$

$\operatorname{Co}(4 d)-\mathrm{N}$ in-plane $\pi^{*}$

Figure S15: Initial CMOs of CMO-SA2-iCAS(10,12) of quintet Co ${ }^{\mathrm{III}}$ (diiminato)(NPh).

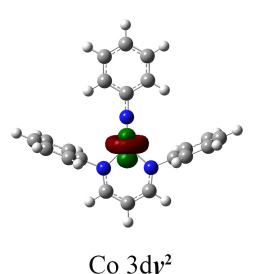

Co $3 \mathrm{~d} y^{2}$

$(1.00)$

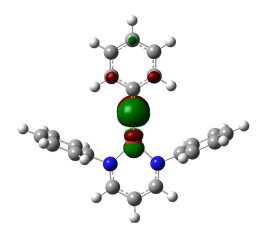

Co(3d)-N out-of-plane $\pi^{*}$ $(1.07)$

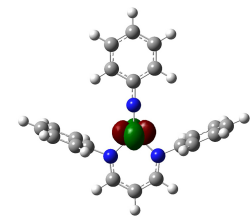

Co $3 \mathrm{~d} x^{2}-z^{2}$

(1.48)

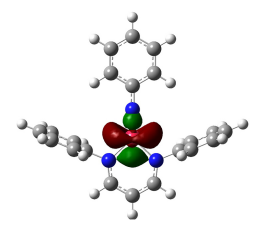

Co $4 \mathrm{~d} y^{2}$

$(0.01)$

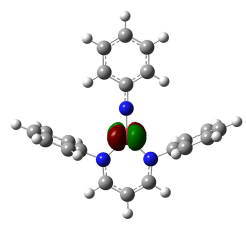

(1.48)
Co $3 \mathrm{~d} x z$

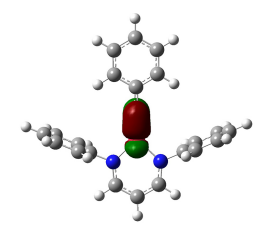

Co-N out-of-plane $\pi$

(1.91)

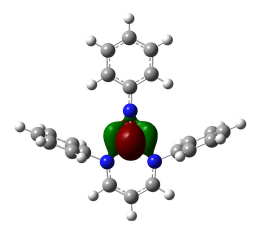

Co $4 \mathrm{~d} x^{2}-z^{2}$

$(0.01)$
Co $4 \mathrm{~d} x z$

$(0.01)$

20.9.

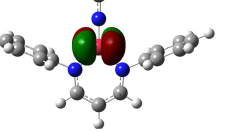

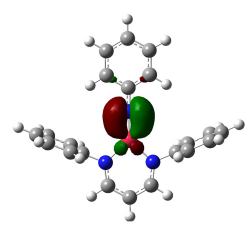

Co-N in-plane $\pi$ (1.92)

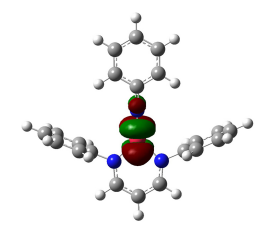

Co(4d)-N out-of-plane $\pi$

(0.01)

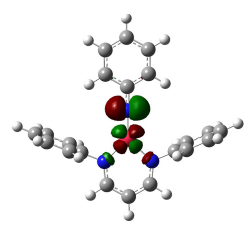

$\mathrm{Co}(3 \mathrm{~d})-\mathrm{N}$ in-plane $\pi^{*}$ (1.07)

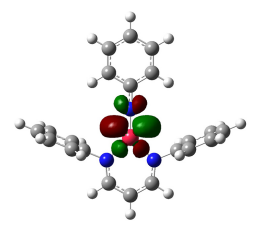

Figure S16: Optimized CMOs of CMO-SA2-iCAS $(10,12)$ of quintet $\mathrm{Co}^{\mathrm{III}}$ (diiminato)(NPh). Occupation numbers are shown in parentheses. 


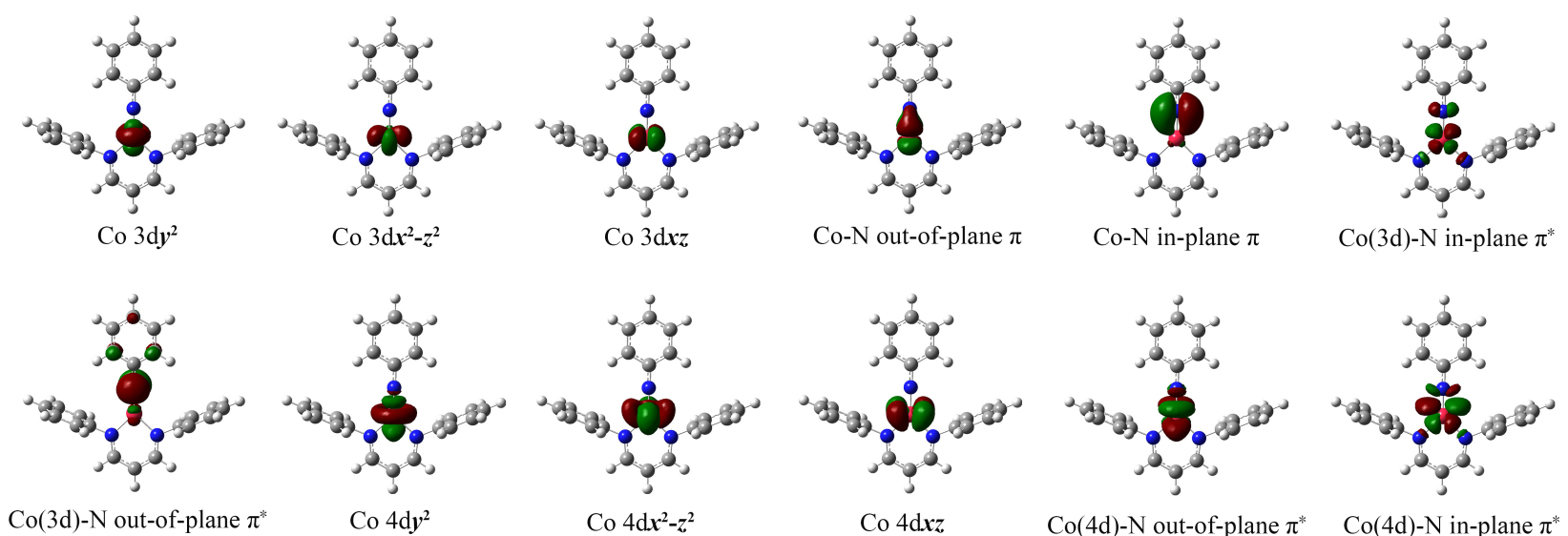

Figure S17: Initial LMOs of LMO-SA2-iCAS $(10,12)$ of quintet $\mathrm{Co}^{\mathrm{III}}$ (diiminato)(NPh).
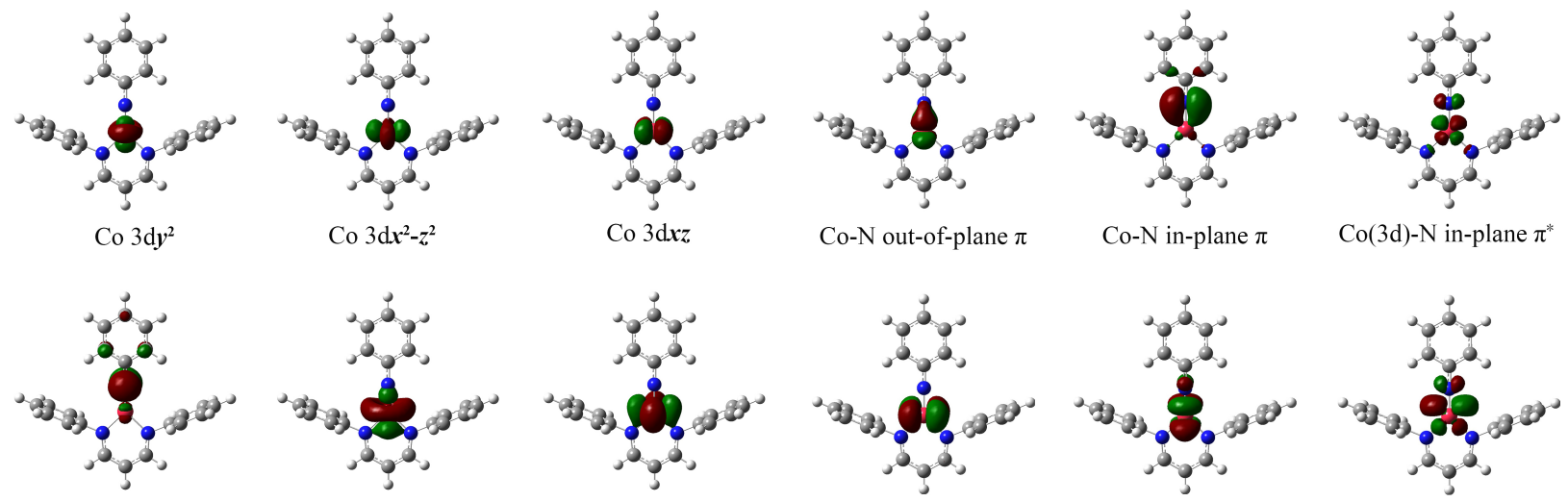

Co-N out-of-plane $\pi$

Co-N in-plane $\pi$

$\operatorname{Co}(3 \mathrm{~d})-\mathrm{N}$ in-plane $\pi^{*}$

$\operatorname{Co}(3 d)-N$ out-of-plane $\pi^{*}$

Co $4 \mathrm{~d} y^{2}$

Co $4 \mathrm{~d} x^{2}-z^{2}$

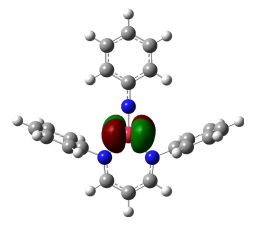

Co $4 \mathrm{~d} x z$

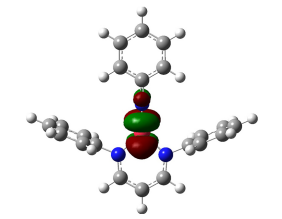

$\operatorname{Co}(4 d)-N$ out-of-plane $\pi^{*}$

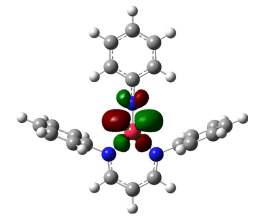

$\mathrm{Co}(4 \mathrm{~d})-\mathrm{N}$ in-plane $\pi^{*}$

Figure S18: Optimized LMOs of LMO-SA2-iCAS $(10,12)$ of quintet $\mathrm{Co}^{\mathrm{III}}$ (diiminato)(NPh). 